\title{
Effects of Protein Supply on Growth Performance, Body Composition and Tissue Deposition for Piglets Fed Diets With or Without Antibiotics
}

\author{
Lei Hou ( $\nabla$ rhoulei@126.com ) \\ Northeast agricultural university \\ Li Wang \\ Guangdong Academy of Agricultural Sciences \\ Cui Zhu \\ Foshan University \\ Xiaolu Wen \\ Guangdong Academy of Agricultural Sciences \\ Hao Xiao \\ Guangdong Academy of Agricultural Sciences \\ Qiwen Wu \\ Guangdong Academy of Agricultural Sciences \\ Zeling Lin \\ South China Agricultural University \\ Zhikang Wang \\ South China Agricultural University \\ Yueqin Qiu \\ Guangdong Academy of Agricultural Sciences \\ Xuefen Yang \\ Guangdong Academy of Agricultural Sciences \\ Kaiguo Gao \\ Guangdong Academy of Agricultural Sciences \\ Zongyong Jiang \\ Guangdong Academy of Agricultural Sciences
}

\section{Research}

Keywords: Antibiotic, Body composition, Crude protein, Growth performance, Piglets, Requirement

Posted Date: August 24th, 2020

DOl: https://doi.org/10.21203/rs.3.rs-63011/v1

License: @ (i) This work is licensed under a Creative Commons Attribution 4.0 International License. Read Full License 


\section{Abstract}

Background: With increasing concerns about antibiotic-resistant bacteria that pose a threat to human and animal health, in-feed antibiotics have been banned since 2006 in Europe and 2020 in China. However, previous studies on crude protein (CP) and amino acids (AA) requirements of pigs were mostly based on the diets with antibiotics. The objective of this study was to evaluate the effects of CP supply on growth performance, body composition and tissue deposition for piglets fed diets with or without antibiotics.

Methods: A total of 250 newly weaned piglets were randomly assigned to 1 of 10 dietary treatments (5 replicate pens per treatment with 5 animals per pen) in a $5 \times 2$ factorial arrangement with $5 \mathrm{CP}$ levels $(16,18,20,22,24 \%)$ and 2 antibiotic supplementations (with or without antibiotics). Treatment diets were fed for $14 \mathrm{~d}$ to test treatment effects, and then a nursery diet (19\% CP) without antibiotics was fed to all piglets until they had an average body weight of $25 \mathrm{~kg}$ to determine if carryover effects of treatment diets existed. An additional 5 piglets at the start were slaughtered to determine initial body composition. At the end of each period, 1 pig close to the average BW of each pen was slaughtered to determine body composition and tissue deposition.

Results: Increasing dietary CP level linearly improved $(P<0.05)$ average daily gain (ADG), average daily feed intake (ADFI), and gain:feed (G:F) during the treatment period, while antibiotics tended to improve ADG of piglets $(P<0.10)$. The ADG during the carryover period tended to be improved $(P<0.10)$ with the increase of dietary CP level, but there was no difference in ADG during the entire nursery period $(P>0.10)$. Neither $\mathrm{CP}$ level nor antibiotic supplementation affected $(P>0.10)$ the incidence of diarrhea in each period. With the increase of dietary CP level, weights of fasted body, empty body, eviscerated carcass, and organs at $d 14$ were linearly improved $(P<0.05)$. Neither CP level nor antibiotic supplementation had a significant effect on the physical body composition of pigs at the end of the nursery period $(P>0.05)$. Body protein content at $\mathrm{d} 14$ linearly improved $(P<0.05)$ with increasing CP level, whereas body ash content, lipid:protein and ash:protein ratio linearly decreased $(P<0.05)$. Increasing dietary CP level resulted in a greater $(P<$ $0.05)$ deposition rates of body water and protein during the treatment period, while antibiotics tended to increase $(P<0.10)$. There was no effect $(P>$ $0.05)$ of $\mathrm{CP}$ level or antibiotic supplementation on the chemical body composition at the end of the nursery period. However, increasing CP level quadratically improved $(P<0.05)$ deposition rates of body water, protein and lipid during the carryover period as well as deposition rates of protein, lipid and ash during the entire nursery period. According to the model with the minimum AIC, the CP requirement based on ADG was 23.01 and $22.65 \%$, and based on protein deposition (PD) was 24.00 and $23.29 \%$ for antibiotic-free diet and antibiotic diet, respectively.

Conclusions: Increasing dietary CP level increased the growth performance and protein deposition of piglets during either the treatment period or the carryover period. The CP requirement for piglets with high health status fed antibiotic-free diet was slightly higher than those fed antibiotic diet.

\section{Background}

Protein nutrition is of paramount importance in newly weaned piglets due to its tremendous capacity for rapid growth and body protein deposition [1]. Previous studies on crude protein (CP) and amino acids (AA) requirements of pigs were mostly based on the diets with antibiotics [2-4]. Antibiotics have played an important role in the control of intestinal disorders and the contribution of growth for a long time [5]. However, with increasing concerns about antibiotic-resistant bacteria that pose a threat to human and animal health, in-feed antibiotics have been banned since 2006 in Europe and 2020 in China [6]. Recent studies have shown that antibiotics can regulate host nitrogen $(\mathrm{N})$ metabolism, partly due to the antibiotic-induced changes in AA fermentation by gut microbiota [7, 8]. In this case, optimal CP requirements for piglets might be different in diets with or without antibiotics. Thus, it is necessary to study the CP requirements after the withdrawal of in-feed antibiotics and to compare their differences with those supplemented with antibiotics.

Besides, it is generally assumed that intestinal disorders of newly weaned piglets are sensitive to CP supply, and the risk of intestinal disorders may be decreased by lowering dietary CP level $[9,10]$. The implementation of this strategy, however, raises the question of whether there is a minimum CP requirement for piglets to allow sufficient $\mathrm{N}$ to be present to generate nonessential AA and to maintain optimal performance [11]. Indeed, studies have shown that a low-CP diet with a balanced AA content can lead to poorer growth performance than conventional diets [12, 13]. Therefore, it is essential to ensure an appropriate CP supply for piglets. However, studies evaluating CP and AA requirement of piglet were usually based on response criteria for production traits such as maximization of average daily gain (ADG) and (or) metabolic traits such as minimization serum urea $\mathrm{N}$ [4, 14, 15], and less on carcass traits such as maximization of body protein deposition (PD). Since the CP requirement for piglet growth depends largely on PD, knowledge of its response to dietary CP supply is more helpful in determining CP requirements. Furthermore, studies have found that protein intake during the suckling period affected growth performance and the proportion of perirenal adipose tissue in the body, and resulted in carryover effects on performance during the post-weaning period [16, 17]. The first two weeks after weaning is a critical period of pig production, but it is unclear whether the CP level and antibiotic supplementation at this time affect the body composition of piglets during the treatment period and at the end of the nursery period.

Therefore, the objective of the current study was to investigate the effects of dietary CP level and antibiotic supplementation on piglet growth, body composition and tissue deposition to find the optimal dietary CP level in newly weaned piglets, and to determine if carryover effects of treatment diets existed.

\section{Materials And Methods}

\section{Animals and experimental design}


A total of 250 newly weaned piglets ( $21 \mathrm{~d}$ of age; $6.39 \pm 0.02 \mathrm{~kg}$ body weight (BW); Duroc $\times$ Landrace $\times$ Yorkshire) were blocked by initial BW and sex and randomly allocated to 50 pens, with 5 animals per pen and 5 replicates per treatment (13 barrows and 12 gilts). Pens were randomly assigned to 1 of 10 dietary treatments in a $5 \times 2$ factorial arrangement with 5 CP levels $(16,18,20,22,24 \%)$ and 2 antibiotic supplementations (with or without antibiotics). The antibiotic supplement used contained $30 \mathrm{mg} / \mathrm{kg}$ bacitracin methylene disalicylate, $75 \mathrm{mg} / \mathrm{kg}$ chlortetracycline and $300 \mathrm{mg} / \mathrm{kg}$ calcium oxytetracycline, and in accordance with the Chinese Regulations of Feeding Drug Additives at the time of the trial. Crystalline AA (Lys, Met, Thr, Trp, Val, and Ile) were supplemented to ensure that the amount of standardized ileal digestibility of essential AA to meet or exceed National Research Council (NRC) recommendations [18]. All diets were formulated to contain similar net energy (NE) contents and were provided in pelleted form. Ingredient composition and nutrient composition of the diets are presented in Tables 1 and 2, respectively. Treatment diets were fed for $14 \mathrm{~d}$, after which all piglets were fed the same nursery diet (19\% CP) without antibiotics until they had an average weight of $25 \mathrm{~kg}$ to determine if carryover effects of treatment diets existed. Piglets were housed in a nursery facility $(2.20 \mathrm{~m} \times 1.50 \mathrm{~m})$, which had a hard plastic fully slatted floor, a multi-hole stainless feeder, and a single bowl drinker. Piglets had free access to feed and water throughout the experiment. 
Table 1

Ingredient composition of experimental diets in weaned piglets (\% as-fed basis)

\begin{tabular}{|c|c|c|c|c|c|c|}
\hline \multirow[t]{2}{*}{ Item } & \multicolumn{5}{|l|}{ d 0 to 14} & \multirow{2}{*}{$\begin{array}{l}\text { d } 14 \text { to end } \\
19 \% \mathrm{CP}\end{array}$} \\
\hline & $16 \% \mathrm{CP}$ & $18 \% \mathrm{CP}$ & $20 \% \mathrm{CP}$ & $22 \% \mathrm{CP}$ & $24 \% \mathrm{CP}$ & \\
\hline \multicolumn{7}{|l|}{ Ingredient } \\
\hline Corn & 45.08 & 38.59 & 34.94 & 31.63 & 28.34 & 68.43 \\
\hline Expanded corn & 10.00 & 10.00 & 10.00 & 10.00 & 10.00 & \\
\hline Expanded soybean & 4.00 & 8.00 & 8.00 & 8.00 & 8.00 & \\
\hline Soybean meal, enzyme treated & 7.92 & 10.00 & 10.00 & 10.00 & 10.00 & 10.00 \\
\hline Soybean meal & & 1.05 & 3.20 & 4.57 & 5.88 & 14.46 \\
\hline Fish meal & 2.00 & 2.00 & 3.20 & 4.57 & 5.88 & 2.00 \\
\hline Whey protein concentrate & 2.00 & 2.00 & 3.20 & 4.57 & 5.88 & \\
\hline Whey powder & 15.00 & 15.00 & 15.00 & 15.00 & 15.00 & \\
\hline Yeast extract & 2.00 & 2.00 & 2.00 & 2.00 & 2.00 & \\
\hline Soybean hulls & 2.00 & 2.00 & 2.00 & 2.00 & 2.00 & \\
\hline Soybean oil & 1.00 & 1.00 & 1.00 & 1.00 & 1.00 & 1.00 \\
\hline Sucrose & 2.50 & 2.50 & 2.50 & 2.50 & 2.50 & \\
\hline Salt & 0.20 & 0.20 & 0.20 & 0.20 & 0.20 & 0.35 \\
\hline Dicalcium phosphate & 1.47 & 1.32 & 1.05 & 0.77 & 0.50 & 0.55 \\
\hline Limestone & & & & & & 0.93 \\
\hline Calcium Citrate & 0.88 & 0.91 & 0.90 & 0.87 & 0.84 & \\
\hline L-Lys HCl & 0.99 & 0.81 & 0.59 & 0.38 & 0.17 & 0.47 \\
\hline DL-Met & 0.18 & 0.15 & 0.10 & 0.05 & 0.01 & 0.06 \\
\hline L-Thr & 0.37 & 0.29 & 0.19 & 0.09 & & 0.12 \\
\hline L-Trp & 0.09 & 0.06 & 0.02 & & & 0.01 \\
\hline L-Val & 0.32 & 0.22 & 0.11 & & & \\
\hline L-Ile & 0.20 & 0.10 & & & & \\
\hline Phytase & 0.02 & 0.02 & 0.02 & 0.02 & 0.02 & 0.02 \\
\hline Zinc oxide & 0.18 & 0.18 & 0.18 & 0.18 & 0.18 & \\
\hline Choline chloride & 0.20 & 0.20 & 0.20 & 0.20 & 0.20 & 0.20 \\
\hline Titanium dioxide & 0.40 & 0.40 & 0.40 & 0.40 & 0.40 & 0.40 \\
\hline Premix $^{1}$ & 1.00 & 1.00 & 1.00 & 1.00 & 1.00 & 1.00 \\
\hline Total & 100.00 & 100.00 & 100.00 & 100.00 & 100.00 & 100.00 \\
\hline $\begin{array}{l}{ }^{1} \text { Provided, per kilogram of diet, } \\
\text { niacin, } 8 \mathrm{mg} \text { pyridoxine, } 40 \mu \mathrm{g} \mathrm{v} \\
0.7 \mathrm{mg} \mathrm{l}, 0.48 \mathrm{mg} \mathrm{Se} .\end{array}$ & $\begin{array}{l}\text { A, } 2800 \\
\text { g biotin, }\end{array}$ & $\begin{array}{l}\min D_{3} \\
\text { g pantott }\end{array}$ & $\begin{array}{l}\text { vitamin } \\
\text { cid, } 1 \mathrm{~ms}\end{array}$ & $\begin{array}{l}\text { g vitamir } \\
\text { acid, } 80\end{array}$ & $\begin{array}{l}\text { mg thian } \\
, 120 \mathrm{mg}\end{array}$ & $\begin{array}{l}\text { boflavin, } 40 \mathrm{n} \\
\text { An, } 16 \mathrm{mg} \mathrm{Cu} \text {, }\end{array}$ \\
\hline
\end{tabular}


Table 2

Nutrient composition of experimental diets in weaned piglets (\% as-fed basis)

\begin{tabular}{|c|c|c|c|c|c|c|}
\hline \multirow[t]{2}{*}{ Item } & \multicolumn{5}{|c|}{ d 0 to 14} & \multirow{2}{*}{$\begin{array}{l}\text { d } 14 \text { to end } \\
19 \% \mathrm{CP}\end{array}$} \\
\hline & $16 \% \mathrm{CP}$ & $18 \% \mathrm{CP}$ & $20 \% \mathrm{CP}$ & $22 \% \mathrm{CP}$ & $24 \% \mathrm{CP}$ & \\
\hline \multicolumn{7}{|c|}{ Calculated composition } \\
\hline $\mathrm{CP}^{1}$ & 16.00 & 17.99 & 19.98 & 21.98 & 23.97 & 18.93 \\
\hline $\mathrm{ME}^{2}, \mathrm{MJ} / \mathrm{kg}$ & 14.32 & 14.42 & 14.47 & 14.55 & 14.64 & 14.08 \\
\hline $\mathrm{NE}^{3}, \mathrm{MJ} / \mathrm{kg}$ & 10.92 & 10.88 & 10.82 & 10.79 & 10.78 & 10.50 \\
\hline Calcium & 0.82 & 0.82 & 0.82 & 0.82 & 0.82 & 0.75 \\
\hline Total phosphorus & 0.72 & 0.72 & 0.72 & 0.72 & 0.72 & 0.61 \\
\hline Total Lys & 1.56 & 1.58 & 1.60 & 1.62 & 1.64 & 1.36 \\
\hline Total Met & 0.45 & 0.46 & 0.46 & 0.47 & 0.47 & 0.40 \\
\hline Total Thr & 0.96 & 0.98 & 1.00 & 1.02 & 1.04 & 0.84 \\
\hline Total Trp & 0.26 & 0.26 & 0.27 & 0.28 & 0.32 & 0.22 \\
\hline Total Val & 0.98 & 1.00 & 1.01 & 1.03 & 1.14 & 0.89 \\
\hline Total Ile & 0.79 & 0.81 & 0.84 & 0.96 & 1.07 & 0.80 \\
\hline Total Leu & 1.28 & 1.45 & 1.64 & 1.84 & 2.02 & 1.69 \\
\hline Total Phe & 0.62 & 0.75 & 0.85 & 0.94 & 1.03 & 0.93 \\
\hline Total Arg & 0.73 & 0.91 & 1.04 & 1.16 & 1.26 & 1.19 \\
\hline Total His & 0.34 & 0.40 & 0.46 & 0.51 & 0.55 & 0.51 \\
\hline SID Lys & 1.41 & 1.41 & 1.41 & 1.41 & 1.41 & 1.23 \\
\hline SID Met & 0.41 & 0.41 & 0.41 & 0.41 & 0.41 & 0.36 \\
\hline SID Thr & 0.83 & 0.83 & 0.83 & 0.83 & 0.84 & 0.73 \\
\hline SID Trp & 0.23 & 0.23 & 0.23 & 0.24 & 0.27 & 0.20 \\
\hline SID Val & 0.89 & 0.89 & 0.89 & 0.89 & 1.00 & 0.78 \\
\hline SID lle & 0.72 & 0.72 & 0.73 & 0.84 & 0.95 & 0.71 \\
\hline SID Leu & 1.13 & 1.27 & 1.45 & 1.63 & 1.80 & 1.51 \\
\hline SID Phe & 0.54 & 0.65 & 0.74 & 0.82 & 0.89 & 0.83 \\
\hline SID Arg & 0.66 & 0.83 & 0.95 & 1.06 & 1.16 & 1.11 \\
\hline SID His & 0.30 & 0.35 & 0.40 & 0.44 & 0.49 & 0.45 \\
\hline SID Lys:NE, g/MJ & 1.29 & 1.30 & 1.31 & 1.31 & 1.31 & 1.17 \\
\hline \multicolumn{7}{|c|}{ Analyzed composition } \\
\hline $\mathrm{CP}^{1}$ & 16.33 & 18.18 & 20.09 & 22.16 & 23.79 & 19.35 \\
\hline Total Lys & 1.51 & 1.51 & 1.52 & 1.53 & 1.55 & 1.24 \\
\hline Total Met & 0.37 & 0.37 & 0.41 & 0.41 & 0.39 & 0.31 \\
\hline Total Thr & 0.95 & 0.97 & 0.99 & 1.01 & 1.03 & 0.79 \\
\hline Total Val & 0.98 & 1.01 & 1.01 & 1.00 & 1.08 & 0.80 \\
\hline Total lle & 0.81 & 0.80 & 0.85 & 0.96 & 1.07 & 0.76 \\
\hline
\end{tabular}

\begin{tabular}{|l|}
\hline${ }^{1} \mathrm{CP}$, crude protein \\
\hline${ }^{2} \mathrm{ME}$, metabolized energy \\
\hline${ }^{2} \mathrm{NE}$, net energy \\
\hline
\end{tabular}




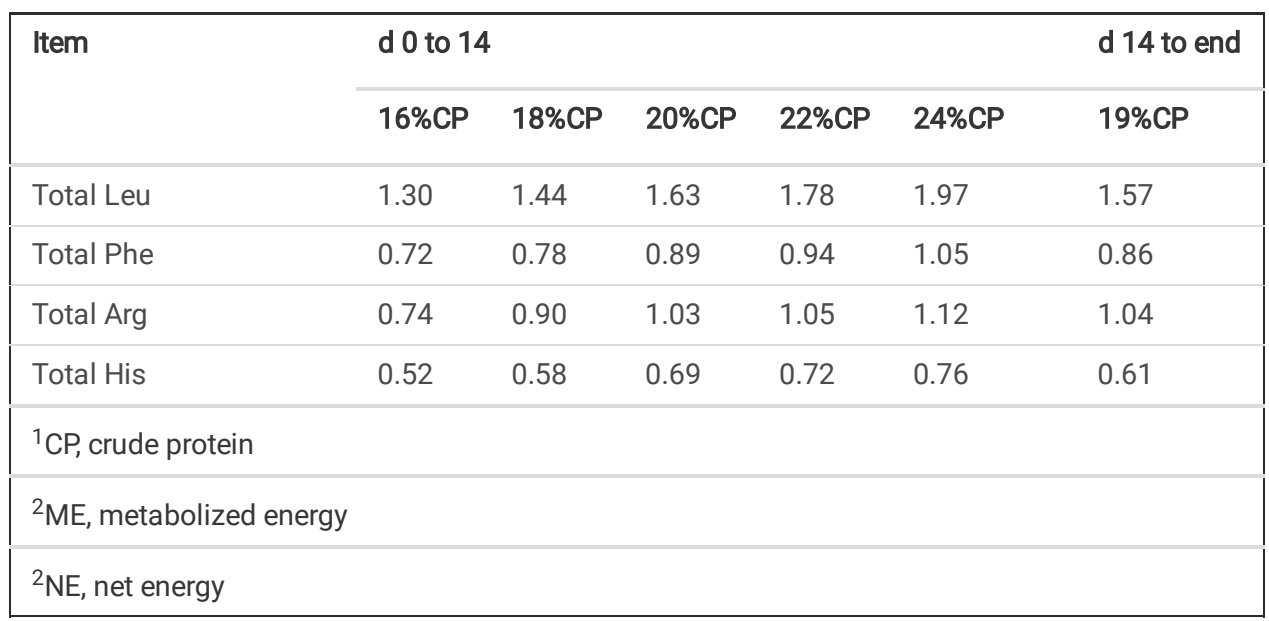

\section{Sample Collection}

Piglets were individually weighed on days 0,14 , and the day when pigs had an average BW of $25 \mathrm{~kg}$, and checked daily for the occurrence of diarrhea. An additional 5 piglets $(6.39 \pm 0.02 \mathrm{~kg} \mathrm{BW} ; 3$ barrows and 2 gilts) at the start were slaughtered as initial slaughter group (ISG) for body composition measurement and assumed to represent the initial body composition of all piglets used in the present study. On day 14 of the experiment and the day when pigs had an average BW of $25 \mathrm{~kg}, 1$ pig close to the average weight of each pen ( 3 barrows and 2 gilts per treatment) was anesthetized with sodium pentobarbital and slaughtered after an overnight fast. Slaughter procedures were performed according to Jones et al. [19]. Blood from each piglet was carefully collected after exsanguination. To obtain the empty body of piglet, the digesta from the stomach and intestines were washed, and the contents from bladder and gallbladder were removed, then they were patted dry. Weights of empty body, organ (including blood, heart, liver, spleen, lungs, kidneys, and stomach and intestines without contents) and eviscerated carcass were recorded. All carcasses were stored at $-20{ }^{\circ} \mathrm{C}$ until further processing. The frozen carcass was cut into small blocks in a double-shaft crusher (L-SP380, LiWill Co. Ltd., Zhengzhou, China), then ground in a grinder with an 18-mm die (SG-130, Yusheng Co., Xingtai, China) and finally ground using an ultrafine grinding mill (GN-130, Yusheng Co., Xingtai, China). The ground carcass was mixed in a mixer for homogenization, and then approximately $2.5 \mathrm{~kg}$ of subsample was taken for chemical analysis using a quartering procedure.

\section{Chemical Analyses}

Both diet and carcass samples were analyzed in duplicate for dry matter, ash, $\mathrm{CP}$, crude fat, and gross energy (GE) according to the methods of $\mathrm{AOAC}$ [20]. The CP content was estimated by multiplying the total $N$ content determined using a Kjeltec 8400 analyzer (FOSS Analytical AB, höganäs, Sweden) by 6.25. Crude fat was determined using an automatic extractor analyzer (XT 15i, Ankom Technology Co., Macedon, NY) and gross energy was measured using a bomb calorimeter (6400, Parr Instrument Co., Moline, IL). Two samples of each diet were hydrolyzed with $6 \mathrm{~mol} / \mathrm{L} \mathrm{HCl}$ at $110{ }^{\circ} \mathrm{C}$ for $24 \mathrm{~h}$, and AA (other than tryptophan) were determined using ion-exchange chromatography with an automatic amino acid analyzer (L-8900, Hitachi Co. Ltd., Tokyo, Japan).

\section{Calculations}

The ADG or average daily feed intake (ADFI) of each pen was calculated as the BW gain or feed consumption during each experimental period divided by the number of pigs and experimental days per pen during the corresponding period. The gain:feed (G:F) was calculated as kilograms of feed consumption divided by the kilogram of BW gain. The incidence of diarrhea was expressed as the percentage of days that pigs with diarrhea to total days observed. Empty BW is the weight of fasted BW after removing the contents of stomach, intestines, gallbladder and bladder. Organs weight is the total weight of blood, heart, liver, spleen, lungs, kidneys, stomach (without digesta) and intestines (without digesta). Eviscerated carcass weight is empty BW minus organs weight. Deposition rates of body water, protein, lipid and ash were calculated as the difference between chemical composition at the end of and the beginning of each experimental period divided by the corresponding experimental days [21].

\section{Statistical analysis}

Data were analyzed using the generalized linear model procedure (Proc GLM) in SAS 9.4 (SAS Institute Inc., Cary, NC) with fixed effects of CP level, antibiotic supplementation, and their interaction. Differences were identified using the least significant difference mean comparison test. Linear and quadratic effects of increasing $\mathrm{CP}$ level were evaluated, and differences in antibiotic supplementation at each CP level were compared by using pairwise comparisons whenever a significant main effect of $\mathrm{CP}$ was observed. Statistical significance was declared at $P<0.05$ and tendencies declared at $P<$ 0.10. Additionally, the optimum dietary CP level and daily N intake for maximal ADG and PD were determined by broken-line and curvilinear-plateau 
models using an NLIN procedure of SAS [22]. If the Hessian produced by the broken-line model is singular, then the analysis of curvilinear-plateau model was not carried out. Evaluation of goodness of fit based on the Akaike information criterion (AIC) and the model with the smallest AIC was select [23].

\section{Results}

\section{Growth performance}

Increasing dietary CP level resulted in heavier BW on d $14(P<0.05)$ due to improved ADG $(P<0.05)$ during the treatment period, while piglets fed the antibiotic diet tended to improve BW and ADG $(P<0.10$; Table 3). The ADG during the carryover period tended to be improved $(P<0.10)$ with the increase of dietary CP level, but there was no difference in ADG during the entire nursery period $(P>0.10)$. The ADFI during each period was improved $(P<0.05)$ by increasing CP level, while the ADFI from d 0 to 14 tended to be increased $(P<0.10)$ with antibiotic supplementation. Increasing dietary CP level increased $\mathrm{G}: \mathrm{F}(P<0.05)$ during the treatment period as well as the entire nursery period. Neither $\mathrm{CP}$ level nor antibiotic supplementation affected $(P>$ $0.10)$ the incidence of diarrhea in each period. There was no interaction between CP level and antibiotic supplementation for any performance variables $(P>0.10)$. Pairwise comparisons of piglets fed diets with or without antibiotics revealed that BW at $d 14, A D G$ and ADFI from d 0 to 14 were increased $(P<0.05)$ at $18 \% \mathrm{CP}$ when the diet supplemented with antibiotics compared to those without antibiotics (Table 4). However, no significant differences $(P>0.05)$ were observed in G:F during the treatment period and the entire nursery period. The BW at $\mathrm{d} 14$ as well as ADG from d 0 to 14 were improved linearly $(P<0.05)$ with increasing CP level. Likewise, G:F from d 0 to 14 and throughout the nursery period were both improved in a linear $(P<0.05)$ manner. Except that ADFI from d 0 to 14 was improved linearly with increasing CP level, ADFI during the carryover period and the entire nursery period quadratically increased $(P<0.05)$. 
Table 3

Effects of CP level and antibiotic supplementation on piglet performance and diarrhea incidence

\begin{tabular}{|c|c|c|c|c|c|c|c|c|c|c|c|c|c|c|}
\hline \multirow[t]{3}{*}{ Item } & \multicolumn{5}{|c|}{ Antibiotic-free diet } & \multicolumn{5}{|c|}{ Antibiotic diet } & \multirow[t]{3}{*}{ SEM } & & & \\
\hline & \multicolumn{10}{|c|}{ CP level, \% } & & \multicolumn{3}{|c|}{$P$-value } \\
\hline & 16 & 18 & 20 & 22 & 24 & 16 & 18 & 20 & 22 & 24 & & $\mathrm{CP}$ & Antibiotic & $\begin{array}{l}\mathrm{CP} \times \\
\text { Antibiotic }\end{array}$ \\
\hline \multicolumn{15}{|l|}{$\mathrm{BW}^{1}, \mathrm{~kg}$} \\
\hline d 0 & 6.38 & 6.39 & 6.39 & 6.39 & 6.4 & 6.39 & 6.39 & 6.39 & 6.39 & 6.39 & 0.02 & 0.98 & 0.97 & 1.00 \\
\hline d 14 & 10.04 & 10.22 & 10.92 & 11.28 & 11.51 & 10.38 & 10.9 & 11.22 & 11.43 & 11.37 & 0.23 & $\begin{array}{l}< \\
0.01\end{array}$ & 0.07 & 0.48 \\
\hline end & 24.49 & 24.6 & 25.17 & 25.55 & 25.33 & 24.52 & 25.08 & 25.23 & 25.66 & 24.88 & 0.42 & 0.13 & 0.86 & 0.86 \\
\hline \multicolumn{15}{|l|}{$A D G^{2}, g$} \\
\hline d 0 to 14 & 261 & 273 & 324 & 349 & 365 & 285 & 322 & 345 & 360 & 356 & 15.8 & $\begin{array}{l}< \\
0.01\end{array}$ & 0.06 & 0.47 \\
\hline $\begin{array}{l}\text { d } 14 \text { to } \\
\text { end }\end{array}$ & 524 & 540 & 538 & 523 & 499 & 497 & 540 & 512 & 538 & 503 & 14.2 & 0.06 & 0.45 & 0.51 \\
\hline d 0 to end & 434 & 449 & 463 & 462 & 454 & 428 & 461 & 454 & 475 & 457 & 13.8 & 0.11 & 0.79 & 0.90 \\
\hline \multicolumn{15}{|l|}{$\mathrm{ADFI}^{3}, \mathrm{~g}$} \\
\hline $\mathrm{d} 0$ to 14 & 372 & 372 & 418 & 433 & 424 & 397 & 417 & 435 & 459 & 420 & 17.6 & 0.01 & 0.06 & 0.74 \\
\hline $\begin{array}{l}d 14 \text { to } \\
\text { end }\end{array}$ & 863 & 886 & 872 & 889 & 819 & 806 & 869 & 873 & 912 & 797 & 23.6 & $\begin{array}{l}< \\
0.01\end{array}$ & 0.35 & 0.54 \\
\hline d 0 to end & 699 & 709 & 716 & 732 & 686 & 671 & 713 & 725 & 756 & 667 & 18.4 & 0.01 & 0.88 & 0.62 \\
\hline \multicolumn{15}{|l|}{$G: F^{4}, g: g$} \\
\hline d 0 to 14 & 0.70 & 0.73 & 0.77 & 0.81 & 0.86 & 0.72 & 0.77 & 0.79 & 0.78 & 0.85 & 0.016 & $\begin{array}{l}< \\
0.01\end{array}$ & 0.47 & 0.28 \\
\hline $\begin{array}{l}\text { d } 14 \text { to } \\
\text { end }\end{array}$ & 0.61 & 0.61 & 0.62 & 0.59 & 0.61 & 0.62 & 0.62 & 0.59 & 0.59 & 0.63 & 0.013 & 0.23 & 0.68 & 0.39 \\
\hline d 0 to end & 0.62 & 0.63 & 0.65 & 0.63 & 0.66 & 0.64 & 0.65 & 0.63 & 0.63 & 0.69 & 0.010 & $\begin{array}{l}< \\
0.01\end{array}$ & 0.36 & 0.21 \\
\hline \multicolumn{15}{|c|}{ Diarrhea incidence, \% } \\
\hline d 0 to 14 & 0.29 & 1.72 & 2.00 & 1.71 & 0.86 & 0.57 & 0.29 & 1.14 & 0.86 & 2.29 & 0.65 & 0.39 & 0.49 & 0.21 \\
\hline $\begin{array}{l}\text { d } 14 \text { to } \\
\text { end }\end{array}$ & 2.61 & 5.25 & 5.33 & 4.97 & 1.95 & 3.02 & 4.76 & 2.74 & 5.08 & 4.28 & 1.06 & 0.13 & 0.95 & 0.25 \\
\hline d 0 to end & 1.56 & 3.59 & 3.76 & 3.46 & 1.45 & 1.94 & 2.67 & 1.98 & 3.08 & 3.35 & 0.68 & 0.18 & 0.71 & 0.10 \\
\hline \multicolumn{15}{|c|}{${ }^{1} \mathrm{BW}$, body weight } \\
\hline \multicolumn{15}{|c|}{${ }^{2} A D G$, average daily gain } \\
\hline \multicolumn{15}{|c|}{${ }^{3} \mathrm{ADFI}$, average daily feed intake } \\
\hline${ }^{4} G: F$, gain & & & & & & & & & & & & & & \\
\hline
\end{tabular}


Table 4

Pairwise comparisons and contrasts of antibiotic supplementation on piglet growth performance

\begin{tabular}{|c|c|c|c|c|c|c|c|}
\hline \multirow[t]{3}{*}{ Item } & \multicolumn{7}{|c|}{ Pairwise comparisons ${ }^{1}$} \\
\hline & \multicolumn{4}{|c|}{ CP level, \% } & \multicolumn{3}{|c|}{ Contrasts } \\
\hline & 16 & 18 & 20 & 22 & 24 & Linear & Quadratic \\
\hline \multicolumn{8}{|l|}{$\mathrm{BW}^{2}, \mathrm{~kg}$} \\
\hline d 14 & 0.40 & 0.03 & 0.37 & 0.64 & 0.68 & $<0.01$ & 0.22 \\
\hline \multicolumn{8}{|l|}{$A D G^{3}, g$} \\
\hline d 0 to 14 & 0.40 & 0.03 & 0.35 & 0.64 & 0.70 & $<0.01$ & 0.21 \\
\hline \multicolumn{8}{|l|}{$\mathrm{ADFI}^{4}, \mathrm{~g}$} \\
\hline d 0 to 14 & 0.38 & 0.04 & 0.50 & 0.36 & 0.90 & $<0.01$ & 0.09 \\
\hline d 14 to end & 0.12 & 0.40 & 0.98 & 0.45 & 0.67 & $<0.01$ & $<0.01$ \\
\hline $\mathrm{d} 0$ to end & 0.29 & 0.75 & 0.72 & 0.33 & 0.63 & $<0.01$ & $<0.01$ \\
\hline \multicolumn{8}{|l|}{$\mathrm{G}: F^{5}, \mathrm{~g}: \mathrm{g}$} \\
\hline d 0 to 14 & 0.55 & 0.18 & 0.26 & 0.25 & 0.63 & $<0.01$ & 0.76 \\
\hline $\mathrm{d} 0$ to end & 0.24 & 0.27 & 0.06 & 0.69 & 0.30 & $<0.01$ & 0.06 \\
\hline \multicolumn{8}{|c|}{${ }^{1}$ Values are the pairwise comparisons between antibiotic-free diet and antibiotic diet at varying $\mathrm{CP}$ leve } \\
\hline \multicolumn{8}{|c|}{${ }^{2} \mathrm{BW}$, body weight } \\
\hline \multicolumn{8}{|c|}{${ }^{3} A D G$, average daily gain } \\
\hline \multicolumn{8}{|c|}{${ }^{4} \mathrm{ADFI}$, average daily feed intake } \\
\hline${ }^{5} \mathrm{G}: F$, gain:fe & & & & & & & \\
\hline
\end{tabular}

\section{Physical Body Composition}

With the increase of dietary CP level, weights of fasted body, empty body, eviscerated carcass, organs, and individual organ including blood, liver, lungs, kidneys and intestines at d 14 were improved $(P<0.05$; Table 5). Antibiotics tended to increase $(P<0.10)$ weights of fasted body, empty body, eviscerated carcass, and lungs. A dietary $\mathrm{CP} \times$ antibiotic interaction $(P<0.05)$ was found for empty body, organs, blood and lungs weights. However, these differences in actual weight did not lead to differences $(P>0.05)$ in relative weight, either in equalizing weight per kg of fasted BW except for eviscerated carcass or in equalizing organ weight per kg of empty BW except for blood. Pairwise comparisons of piglets fed diets with or without antibiotics revealed that antibiotics increased $(P<0.05)$ weights of fasted body, empty body, eviscerated carcass and blood on d 14 at $18 \% \mathrm{CP}($ Table 6$)$. The weight of physical body composition was improved linearly $(P<0.05)$ with increasing CP level. Neither CP level nor antibiotic supplementation had a significant effect on the physical body composition of pigs at the end of the nursery period $(P>0.05 ;$ Table 7$)$. 
Table 5

Effects of CP level and antibiotic supplementation on the physical body composition of piglets at d 14 after weaning

\begin{tabular}{|c|c|c|c|c|c|c|c|c|c|c|c|c|c|c|}
\hline \multirow[t]{3}{*}{ Item ${ }^{1}$} & \multicolumn{5}{|c|}{ Antibiotic-free diet } & \multicolumn{5}{|c|}{ Antibiotic diet } & \multirow[t]{3}{*}{ SEM } & & & \\
\hline & \multicolumn{10}{|c|}{ CP level, \% } & & \multicolumn{3}{|c|}{$P$-value } \\
\hline & 16 & 18 & 20 & 22 & 24 & 16 & 18 & 20 & 22 & 24 & & $\mathrm{CP}$ & Antibiotic & $\begin{array}{l}\mathrm{CP} x \\
\text { Antibiotic }\end{array}$ \\
\hline \multicolumn{15}{|l|}{ Weight, kg } \\
\hline Fasted BW & 9.83 & 9.96 & 10.63 & 11.18 & 11.47 & 10.19 & 10.72 & 11.00 & 11.29 & 11.13 & 0.20 & $\begin{array}{l}< \\
0.01\end{array}$ & 0.06 & 0.16 \\
\hline $\begin{array}{l}\text { Eviscerated } \\
\text { carcass }\end{array}$ & 8.03 & 8.00 & 8.52 & 9.04 & 9.24 & 8.39 & 8.72 & 8.84 & 8.93 & 8.97 & 0.18 & $\begin{array}{l}< \\
0.01\end{array}$ & 0.09 & 0.09 \\
\hline $\operatorname{Organ}^{1}$ & 1.56 & 1.60 & 1.76 & 1.81 & 1.91 & 1.57 & 1.73 & 1.82 & 1.90 & 1.79 & 0.04 & $\begin{array}{l}< \\
0.01\end{array}$ & 0.16 & 0.04 \\
\hline Empty BW ${ }^{2}$ & 9.59 & 9.60 & 10.28 & 10.85 & 11.15 & 9.96 & 10.45 & 10.66 & 10.83 & 10.76 & 0.19 & $\begin{array}{l}<.01 \\
0.01\end{array}$ & 0.06 & 0.04 \\
\hline \multicolumn{15}{|c|}{ Weight, $\mathrm{g} / \mathrm{kg}$ fasted BW } \\
\hline $\begin{array}{l}\text { Eviscerated } \\
\text { carcass }\end{array}$ & 817 & 803 & 802 & 808 & 806 & 823 & 814 & 803 & 791 & 805 & 5.3 & $\begin{array}{l}< \\
0.01\end{array}$ & 0.93 & 0.10 \\
\hline Organ $^{1}$ & 159 & 161 & 166 & 162 & 167 & 154 & 162 & 165 & 168 & 162 & 4.3 & 0.21 & 0.87 & 0.67 \\
\hline Empty BW ${ }^{2}$ & 976 & 964 & 967 & 970 & 972 & 978 & 976 & 968 & 959 & 967 & 6.1 & 0.36 & 0.97 & 0.40 \\
\hline \multicolumn{15}{|c|}{ Organ weight, g } \\
\hline Blood & 467 & 430 & 521 & 563 & 566 & 446 & 506 & 548 & 553 & 525 & 18.6 & $\begin{array}{l}< \\
0.01\end{array}$ & 0.60 & 0.03 \\
\hline Heart & 54 & 60 & 61 & 58 & 59 & 62 & 62 & 56 & 63 & 67 & 3.8 & 0.67 & 0.15 & 0.39 \\
\hline Liver & 231 & 236 & 271 & 267 & 305 & 224 & 271 & 257 & 283 & 268 & 12.2 & $\begin{array}{l}<.01 \\
0.01\end{array}$ & 0.87 & 0.06 \\
\hline Spleen & 22 & 21 & 23 & 20 & 28 & 25 & 21 & 24 & 29 & 25 & 2.1 & 0.14 & 0.18 & 0.08 \\
\hline Lungs & 106 & 116 & 115 & 116 & 144 & 116 & 125 & 134 & 133 & 122 & 6.1 & 0.02 & 0.10 & 0.02 \\
\hline Kidneys & 63 & 71 & 76 & 71 & 84 & 67 & 75 & 72 & 78 & 80 & 4.4 & 0.01 & 0.65 & 0.61 \\
\hline Stomach ${ }^{3}$ & 61 & 63 & 63 & 64 & 66 & 61 & 68 & 66 & 66 & 66 & 2.9 & 0.43 & 0.37 & 0.92 \\
\hline Intestines 4 & 558 & 602 & 628 & 649 & 655 & 572 & 604 & 659 & 695 & 642 & 28.4 & $\begin{array}{l}<.01 \\
0.01\end{array}$ & 0.38 & 0.87 \\
\hline \multicolumn{15}{|c|}{ Organ weight, g/kg empty BW } \\
\hline Blood & 48.6 & 44.8 & 50.7 & 51.9 & 50.8 & 44.8 & 48.5 & 51.5 & 51.1 & 48.9 & 1.71 & 0.01 & 0.71 & 0.26 \\
\hline Heart & 5.6 & 6.3 & 5.9 & 5.4 & 5.3 & 6.2 & 5.9 & 5.3 & 5.8 & 6.2 & 0.35 & 0.57 & 0.39 & 0.16 \\
\hline Liver & 24.1 & 24.5 & 26.5 & 24.6 & 27.3 & 22.5 & 26.0 & 24.1 & 26.2 & 25.0 & 1.24 & 0.23 & 0.41 & 0.31 \\
\hline Spleen & 2.3 & 2.2 & 2.2 & 1.9 & 2.5 & 2.5 & 2.0 & 2.3 & 2.7 & 2.3 & 0.19 & 0.47 & 0.32 & 0.06 \\
\hline Lungs & 11.1 & 12.1 & 11.2 & 10.7 & 12.9 & 11.6 & 12.0 & 12.6 & 12.3 & 11.3 & 0.56 & 0.60 & 0.31 & 0.07 \\
\hline Kidneys & 6.5 & 7.4 & 7.4 & 6.6 & 7.6 & 6.8 & 7.2 & 6.8 & 7.2 & 7.4 & 0.42 & 0.30 & 0.90 & 0.61 \\
\hline Stomach ${ }^{3}$ & 6.4 & 6.6 & 6.2 & 5.9 & 5.9 & 6.1 & 6.5 & 6.2 & 6.1 & 6.1 & 0.30 & 0.39 & 1.00 & 0.92 \\
\hline Intestines 4 & 58.4 & 62.7 & 61.1 & 60.0 & 58.7 & 57.4 & 57.8 & 61.9 & 64.3 & 59.8 & 2.81 & 0.55 & 0.97 & 0.60 \\
\hline \multicolumn{15}{|c|}{${ }^{1}$ Organs weight is the total weight of blood, heart, liver, spleen, lungs, kidneys, stomach (without digesta) and intestines (without digesta) } \\
\hline \multicolumn{15}{|c|}{${ }^{2} E m p t y ~ B W$ is the sum of eviscerated carcass weight and organs weight } \\
\hline \multicolumn{15}{|c|}{${ }^{3}$ Stomach, without digesta } \\
\hline
\end{tabular}


Table 6

Pairwise comparisons and contrasts of antibiotic supplementation on the physical body composition of piglets at $\mathrm{d} 14$ after weaning

\begin{tabular}{|c|c|c|c|c|c|c|c|}
\hline \multirow[t]{3}{*}{ Item } & \multicolumn{7}{|c|}{ Pairwise comparisons ${ }^{1}$} \\
\hline & \multicolumn{5}{|c|}{ CP level, \% } & \multicolumn{2}{|c|}{ Contrasts } \\
\hline & 16 & 18 & 20 & 22 & 24 & Linear & Quadratic \\
\hline \multicolumn{8}{|l|}{ Weight, kg } \\
\hline Fasted BW & 0.34 & 0.01 & 0.12 & 0.74 & 0.29 & $<0.01$ & 0.37 \\
\hline Eviscerated carcass & 0.27 & $<0.01$ & 0.19 & 0.73 & 0.31 & $<0.01$ & 0.94 \\
\hline Organ $^{2}$ & 0.86 & 0.11 & 0.26 & 0.06 & 0.06 & $<0.01$ & 0.12 \\
\hline Empty $\mathrm{BW}^{3}$ & 0.31 & $<0.01$ & 0.15 & 0.95 & 0.18 & $<0.01$ & 0.64 \\
\hline \multicolumn{8}{|c|}{ Weight, $\mathrm{g} / \mathrm{kg}$ fasted BW } \\
\hline Eviscerated carcass & 0.31 & 0.25 & 0.84 & 0.06 & 0.96 & 0.01 & 0.01 \\
\hline \multicolumn{8}{|l|}{ Organ weight, g } \\
\hline Blood & 0.50 & 0.01 & 0.38 & 0.69 & 0.16 & $<0.01$ & 0.13 \\
\hline Liver & 0.61 & 0.25 & 0.30 & 0.07 & 0.10 & $<0.01$ & 0.52 \\
\hline Lungs & 0.24 & 0.39 & 0.01 & 0.08 & 0.08 & $<0.01$ & 0.93 \\
\hline Kidneys & 0.49 & 0.56 & 0.55 & 0.37 & 0.37 & $<0.01$ & 0.90 \\
\hline Intestines ${ }^{4}$ & 0.64 & 0.97 & 0.52 & 0.09 & 0.81 & $<0.01$ & 0.08 \\
\hline \multicolumn{8}{|c|}{ Organ weight, g/kg empty BW } \\
\hline Blood & 0.08 & 0.10 & 0.81 & 0.70 & 0.54 & 0.04 & 0.21 \\
\hline \multicolumn{8}{|c|}{${ }^{1}$ Values are the pairwise comparisons between antibiotic-free diet and antibiotic diet at varying $\mathrm{CP}$ levels } \\
\hline \multicolumn{8}{|c|}{${ }^{2}$ Organs weight is the total weight of blood, heart, liver, spleen, lungs, kidneys, stomach (without digesta) and intestines (without $d$} \\
\hline
\end{tabular}


Table 7

Effects of CP level and antibiotic supplementation on the physical body composition of piglets at the end of the nursery period

\begin{tabular}{|c|c|c|c|c|c|c|c|c|c|c|c|c|c|c|}
\hline \multirow{3}{*}{ Item $^{1}$} & \multicolumn{5}{|c|}{ Antibiotic-free diet } & \multicolumn{5}{|c|}{ Antibiotic diet } & \multirow[t]{3}{*}{ SEM } & & & \\
\hline & \multicolumn{10}{|c|}{ CP level, \% } & & \multicolumn{3}{|c|}{$P$ value } \\
\hline & 16 & 18 & 20 & 22 & 24 & 16 & 18 & 20 & 22 & 24 & & $\mathrm{CP}$ & Antibiotic & $\begin{array}{l}\text { CP } \times \\
\text { Antibiotic }\end{array}$ \\
\hline \multicolumn{15}{|l|}{ Weight, kg } \\
\hline Fasted BW & 24.45 & 24.97 & 24.86 & 25.47 & 25.57 & 24.2 & 24.99 & 25.18 & 25.28 & 24.71 & 0.85 & 0.79 & 0.73 & 0.97 \\
\hline $\begin{array}{l}\text { Eviscerated } \\
\text { carcass }\end{array}$ & 19.81 & 20.42 & 20.53 & 21.03 & 21.15 & 19.99 & 20.35 & 20.72 & 20.78 & 20.15 & 0.75 & 0.72 & 0.70 & 0.93 \\
\hline $\operatorname{Organ}^{1}$ & 3.81 & 3.79 & 3.7 & 3.82 & 3.79 & 3.6 & 3.83 & 3.8 & 3.96 & 3.88 & 0.14 & 0.71 & 0.73 & 0.71 \\
\hline Empty BW² & 23.62 & 24.21 & 24.23 & 24.85 & 24.94 & 23.59 & 24.18 & 24.52 & 24.74 & 24.03 & 0.81 & 0.67 & 0.76 & 0.96 \\
\hline \multicolumn{15}{|c|}{ Weight, $\mathrm{g} / \mathrm{kg}$ fasted BW } \\
\hline $\begin{array}{l}\text { Eviscerated } \\
\text { carcass }\end{array}$ & 811 & 817 & 826 & 826 & 827 & 826 & 814 & 823 & 822 & 814 & 7.7 & 0.73 & 0.74 & 0.48 \\
\hline Organ $^{1}$ & 156 & 152 & 149 & 150 & 149 & 149 & 154 & 151 & 156 & 159 & 5.6 & 0.95 & 0.46 & 0.63 \\
\hline Empty BW ${ }^{2}$ & 967 & 969 & 975 & 976 & 975 & 975 & 967 & 974 & 979 & 973 & 4.9 & 0.47 & 0.74 & 0.79 \\
\hline \multicolumn{15}{|c|}{ Organ weight, g } \\
\hline Blood & 1124 & 1123 & 1021 & 1134 & 1160 & 1073 & 1143 & 1086 & 1272 & 1164 & 53.4 & 0.07 & 0.31 & 0.49 \\
\hline Heart & 110 & 121 & 129 & 124 & 122 & 120 & 112 & 113 & 112 & 115 & 6.8 & 0.90 & 0.12 & 0.34 \\
\hline Liver & 564 & 586 & 586 & 606 & 567 & 516 & 552 & 592 & 594 & 581 & 39.1 & 0.60 & 0.55 & 0.92 \\
\hline Spleen & 65 & 63 & 75 & 77 & 49 & 55 & 52 & 59 & 64 & 56 & 7.2 & 0.13 & 0.07 & 0.56 \\
\hline Lungs & 272 & 250 & 297 & 247 & 234 & 238 & 275 & 253 & 259 & 264 & 20.8 & 0.71 & 0.89 & 0.25 \\
\hline Kidneys & 124 & 125 & 134 & 138 & 126 & 133 & 139 & 130 & 145 & 130 & 9.6 & 0.66 & 0.33 & 0.91 \\
\hline Stomach ${ }^{3}$ & 143 & 153 & 136 & 146 & 138 & 139 & 146 & 147 & 134 & 147 & 8.3 & 0.83 & 0.95 & 0.56 \\
\hline Intestines ${ }^{4}$ & 1390 & 1345 & 1296 & 1315 & 1371 & 1304 & 1391 & 1391 & 1357 & 1395 & 59.9 & 0.94 & 0.53 & 0.60 \\
\hline \multicolumn{15}{|c|}{ Organ weight, g/kg empty BW } \\
\hline Blood & 58.9 & 55.7 & 53.6 & 53.0 & 55.1 & 55.5 & 57.8 & 56.8 & 54.8 & 58.8 & 3.06 & 0.80 & 0.45 & 0.76 \\
\hline Heart & 4.7 & 5.0 & 5.4 & 5.0 & 4.9 & 5.1 & 4.7 & 4.6 & 4.6 & 4.8 & 0.24 & 0.92 & 0.12 & 0.15 \\
\hline Liver & 23.9 & 24.2 & 24.2 & 24.4 & 22.8 & 21.9 & 22.8 & 24.0 & 24.0 & 24.3 & 1.41 & 0.88 & 0.60 & 0.79 \\
\hline Spleen & 2.7 & 2.6 & 3.1 & 3.1 & 2.0 & 2.3 & 2.1 & 2.4 & 2.6 & 2.3 & 0.26 & 0.10 & 0.05 & 0.38 \\
\hline Lungs & 11.4 & 10.4 & 12.2 & 10.0 & 9.4 & 10.1 & 11.4 & 10.3 & 10.5 & 11.0 & 0.73 & 0.55 & 1.00 & 0.08 \\
\hline Kidneys & 5.2 & 5.2 & 5.5 & 5.6 & 5.1 & 5.6 & 5.8 & 5.3 & 5.9 & 5.5 & 0.33 & 0.75 & 0.17 & 0.75 \\
\hline Stomach ${ }^{3}$ & 6.1 & 6.3 & 5.6 & 5.9 & 5.6 & 5.9 & 6.1 & 6.0 & 5.4 & 6.1 & 0.24 & 0.26 & 0.94 & 0.17 \\
\hline Intestines ${ }^{4}$ & 58.9 & 55.7 & 53.6 & 53.0 & 55.1 & 55.5 & 57.8 & 56.8 & 54.8 & 58.8 & 3.06 & 0.80 & 0.46 & 0.76 \\
\hline \multicolumn{15}{|c|}{${ }^{1}$ Organs weight is the total weight of blood, heart, liver, spleen, lungs, kidneys, stomach (without digesta) and intestines (without digesta) } \\
\hline \multicolumn{15}{|c|}{${ }^{2}$ Empty BW is the sum of eviscerated carcass weight and organs weight } \\
\hline \multicolumn{15}{|c|}{${ }^{3}$ Stomach, without digesta } \\
\hline
\end{tabular}

\section{Chemical Body Composition}


Body protein content at d 14 was improved $(P<0.05)$ with increasing CP level, whereas body ash content, lipid:protein and ash:protein ratio decreased $(P<0.05$; Table 8). A dietary CP $\times$ antibiotic interaction $(P<0.05)$ was observed on body protein content and water:protein ratio at $\mathrm{d} 14$. Increasing dietary $\mathrm{CP}$ level resulted in a greater $(P<0.05)$ deposition rates of body water and protein during the treatment period, while antibiotics tended to increase $(P<0.10)$. Deposition rates of lipid and ash during the treatment period also changed $(P<0.05)$ with dietary $\mathrm{CP}$ level. There was a significant $(P<0.05)$ interaction between dietary $\mathrm{CP}$ and antibiotics for PD rate during the treatment period. Pairwise comparisons of piglets fed diets with or without antibiotics revealed that antibiotics decreased $(P<0.05)$ body lipid:protein ratio at $18 \%$ CP, tended to decrease $(P<0.01)$ body protein content and body ash:protein ratio at $24 \%$ and $16 \% \mathrm{CP}$, respectively (Table 9$)$. Body protein content was increased linearly $(P<0.05)$ with increasing $\mathrm{CP}$ level, whereas body ash content, body lipid:protein and ash:protein ratio were decreased linearly $(P<0.05)$. Antibiotics increased $(P<0.05) \mathrm{PD}$ rate at $18 \% \mathrm{CP}$ and decreased $(P<0.05)$ body lipid deposition rate at $24 \%$ CP during the treatment period. Deposition rates of body water and protein during the treatment period were increased linearly $(P<0.05)$ with increasing CP level, while body ash deposition rate was increased quadratically $(P<0.05)$.

Table 8

Effects of CP level and antibiotic supplementation on the chemical body composition of piglets during the treatment period

\begin{tabular}{|c|c|c|c|c|c|c|c|c|c|c|c|c|c|c|c|}
\hline \multirow[t]{3}{*}{ Item ${ }^{1}$} & \multicolumn{6}{|c|}{ Antibiotic-free diet } & \multicolumn{5}{|c|}{ Antibiotic diet } & \multirow[t]{3}{*}{ SEM } & & & \\
\hline & \multirow[b]{2}{*}{$\mathrm{ISG}^{1}$} & \multicolumn{10}{|c|}{ CP level, \% } & & \multicolumn{3}{|c|}{$P$ value } \\
\hline & & 16 & 18 & 20 & 22 & 24 & 16 & 18 & 20 & 22 & 24 & & $\mathrm{CP}$ & Antibiotic & $\begin{array}{l}\mathrm{CP} \times \\
\text { Antibiotic }\end{array}$ \\
\hline \multicolumn{16}{|c|}{ Chemical composition } \\
\hline Water, \% & 69.7 & 70.8 & 70.5 & 70.9 & 70.8 & 70.6 & 70.0 & 69.8 & 71.1 & 70.9 & 71.6 & 0.55 & 0.39 & 0.91 & 0.51 \\
\hline Protein, \% & 15.6 & 15.7 & 15.6 & 16.3 & 16.0 & 16.6 & 16.0 & 16.1 & 16.0 & 15.9 & 16.1 & 0.17 & 0.03 & 0.89 & 0.03 \\
\hline Lipid, \% & 10.8 & 9.7 & 10.7 & 9.0 & 9.5 & 9.2 & 10.0 & 10.1 & 9.0 & 9.3 & 8.7 & 0.55 & 0.07 & 0.58 & 0.94 \\
\hline Ash, \% & 3.0 & 3.1 & 3.1 & 3.1 & 3.0 & 2.8 & 3.0 & 3.0 & 3.1 & 2.9 & 2.8 & 0.09 & 0.03 & 0.46 & 0.89 \\
\hline $\mathrm{GE}^{2}, \mathrm{MJ} / \mathrm{kg}$ & 8.1 & 7.6 & 7.9 & 7.6 & 7.6 & 7.6 & 7.9 & 8.0 & 7.5 & 7.6 & 7.3 & 0.21 & 0.22 & 0.93 & 0.66 \\
\hline Water:protein & 4.5 & 4.5 & 4.5 & 4.4 & 4.4 & 4.3 & 4.4 & 4.4 & 4.5 & 4.5 & 4.5 & 0.07 & 0.63 & 0.97 & 0.03 \\
\hline Lipid:protein & 0.69 & 0.62 & 0.69 & 0.55 & 0.59 & 0.55 & 0.62 & 0.63 & 0.57 & 0.59 & 0.54 & 0.036 & 0.03 & 0.60 & 0.88 \\
\hline Ash:protein & 0.19 & 0.20 & 0.20 & 0.19 & 0.19 & 0.17 & 0.19 & 0.19 & 0.19 & 0.18 & 0.18 & 0.007 & 0.01 & 0.27 & 0.43 \\
\hline \multicolumn{16}{|c|}{ Deposition rate } \\
\hline \multicolumn{16}{|l|}{ d 0 to 14} \\
\hline Water, g/d & & 190 & 197 & 235 & 252 & 262 & 201 & 226 & 252 & 260 & 264 & 11.2 & $\dot{0.01}$ & 0.07 & 0.79 \\
\hline Protein, g/d & & 41 & 42 & 56 & 58 & 65 & 48 & 54 & 57 & 58 & 59 & 2.5 & $\begin{array}{l}<.01 \\
0.01\end{array}$ & 0.09 & 0.02 \\
\hline Lipid, g/d & & 21 & 28 & 21 & 27 & 26 & 25 & 29 & 23 & 27 & 21 & 1.5 & $\begin{array}{l}<.01 \\
0.01\end{array}$ & 0.72 & 0.06 \\
\hline Ash, g/d & & 8.6 & 8.5 & 10.3 & 10.3 & 9.1 & 8.6 & 9.5 & 11.2 & 9.7 & 9.1 & 0.48 & $\begin{array}{l}<.01 \\
0.01\end{array}$ & 0.41 & 0.43 \\
\hline \multicolumn{16}{|c|}{${ }^{1}$ ISG, initial slaughter group } \\
\hline
\end{tabular}


Table 9

Pairwise comparisons and contrasts of antibiotic supplementation on the chemical body composition of piglets during the treatment period

\begin{tabular}{|c|c|c|c|c|c|c|c|}
\hline \multirow[t]{3}{*}{ Item $^{1}$} & \multicolumn{7}{|c|}{ Pairwise comparisons ${ }^{1}$} \\
\hline & \multicolumn{5}{|c|}{ CP level, \% } & \multicolumn{2}{|c|}{ Contrasts } \\
\hline & 16 & 18 & 20 & 22 & 24 & Linear & Quadratic \\
\hline \multicolumn{8}{|c|}{ Chemical composition } \\
\hline Protein, \% & 0.21 & 0.16 & 0.14 & 0.47 & 0.06 & 0.01 & 0.35 \\
\hline Ash, \% & 0.15 & 0.55 & 0.91 & 0.32 & 0.74 & 0.01 & 0.11 \\
\hline Lipid:protein & 1.00 & 0.05 & 0.71 & 0.95 & 0.81 & 0.01 & 0.79 \\
\hline Ash:protein & 0.05 & 0.27 & 0.94 & 0.21 & 0.26 & $<0.01$ & 0.15 \\
\hline \multicolumn{8}{|c|}{ Deposition rate, $\mathrm{g} / \mathrm{d}$} \\
\hline \multicolumn{8}{|l|}{ d 0 to 14} \\
\hline Water & 0.58 & 0.05 & 0.31 & 0.60 & 0.93 & $<0.01$ & 0.21 \\
\hline Protein & 0.16 & $<0.01$ & 0.78 & 0.88 & 0.16 & $<0.01$ & 0.53 \\
\hline Lipid & 0.17 & 0.68 & 0.35 & 0.95 & 0.04 & 0.93 & 0.19 \\
\hline Ash & 0.95 & 0.12 & 0.21 & 0.37 & 0.96 & $<0.01$ & $<0.01$ \\
\hline
\end{tabular}

There was no effect $(P>0.05)$ of $\mathrm{CP}$ level or antibiotic supplementation on the chemical body composition at the end of the nursery period (Table 10$)$. However, increasing dietary CP level improved $(P<0.05)$ deposition rates of body water, protein and lipid during the carryover period as well as deposition rates of protein, lipid and ash during the entire nursery period. There was a significant $(P<0.05)$ interaction between dietary $\mathrm{CP}$ and antibiotics for lipid and ash deposition rates during the carryover period and lipid deposition rate during the entire nursery period. Pairwise comparisons of piglets fed diets with or without antibiotics revealed that body lipid deposition rate for pigs fed with antibiotic diet was increased $(P<0.05)$ at $24 \% \mathrm{CP}$, but decreased $(P<0.05)$ at $18 \% \mathrm{CP}$ both during the carryover period and the entire nursery period, and also decreased $(P<0.05)$ at $16 \% \mathrm{CP}$ during the carryover period (Table 11). The decline $(P<0.05)$ in water and protein deposition rates coupled with increased $(P<0.05)$ lipid deposition rate during the carryover period were observed at $20 \% \mathrm{CP}$ when piglets fed diets with antibiotics. The body composition deposition variables during the carryover period and the entire nursery period both increased quadratically $(P<0.05)$ as the CP level increased. 
Table 10

Effects of CP level and antibiotic supplementation on the chemical body composition of piglets during the carryover period

\begin{tabular}{|c|c|c|c|c|c|c|c|c|c|c|c|c|c|c|}
\hline \multirow[t]{3}{*}{ Item ${ }^{1}$} & \multicolumn{5}{|c|}{ Antibiotic-free diet } & \multicolumn{5}{|c|}{ Antibiotic diet } & \multirow[t]{3}{*}{ SEM } & & & \\
\hline & \multicolumn{10}{|c|}{ CP level, \% } & & \multicolumn{3}{|c|}{$P$ value } \\
\hline & 16 & 18 & 20 & 22 & 24 & 16 & 18 & 20 & 22 & 24 & & $\mathrm{CP}$ & Antibiotic & $\mathrm{CP} \times$ Antibiotic \\
\hline \multicolumn{15}{|c|}{ Chemical composition } \\
\hline Water, \% & 68.4 & 69.0 & 69.2 & 68.1 & 69.8 & 68.9 & 70.2 & 68.5 & 68.4 & 69.0 & 0.51 & 0.09 & 0.76 & 0.25 \\
\hline Protein, \% & 16.5 & 16.5 & 16.8 & 16.6 & 16.9 & 16.6 & 16.5 & 16.6 & 16.7 & 16.3 & 0.20 & 0.87 & 0.32 & 0.39 \\
\hline Lipid, \% & 11.3 & 11.2 & 10.9 & 11.7 & 10.0 & 11.0 & 9.9 & 11.7 & 11.1 & 11.3 & 0.59 & 0.50 & 0.87 & 0.21 \\
\hline Ash, \% & 2.7 & 2.7 & 2.8 & 2.8 & 2.6 & 2.7 & 2.7 & 2.8 & 2.6 & 2.8 & 0.09 & 0.63 & 0.85 & 0.55 \\
\hline $\mathrm{GE}^{1}, \mathrm{MJ} / \mathrm{kg}$ & 8.6 & 8.4 & 8.3 & 8.8 & 8.0 & 8.4 & 7.9 & 8.6 & 8.5 & 8.4 & 0.21 & 0.17 & 0.61 & 0.17 \\
\hline Water:protein & 4.1 & 4.2 & 4.1 & 4.1 & 4.1 & 4.2 & 4.3 & 4.1 & 4.1 & 4.2 & 0.05 & 0.19 & 0.26 & 0.73 \\
\hline Lipid:protein & 0.69 & 0.68 & 0.65 & 0.71 & 0.59 & 0.66 & 0.61 & 0.70 & 0.67 & 0.64 & 0.041 & 0.45 & 0.82 & 0.50 \\
\hline Ash:protein & 0.17 & 0.16 & 0.17 & 0.17 & 0.16 & 0.16 & 0.17 & 0.17 & 0.16 & 0.17 & 0.007 & 0.91 & 0.38 & 0.24 \\
\hline \multicolumn{15}{|c|}{ Deposition rate, $\mathrm{g} / \mathrm{d}$} \\
\hline \multicolumn{15}{|l|}{ d 14 to end } \\
\hline Water & 350 & 367 & 365 & 353 & 345 & 339 & 380 & 340 & 358 & 335 & 9.6 & 0.01 & 0.35 & 0.34 \\
\hline Protein & 90 & 93 & 92 & 92 & 86 & 85 & 90 & 88 & 94 & 83 & 2.4 & 0.01 & 0.10 & 0.57 \\
\hline Lipid & 65 & 63 & 67 & 72 & 53 & 58 & 53 & 71 & 67 & 68 & 1.8 & $<0.01$ & 0.53 & $<0.01$ \\
\hline Ash & 13 & 13 & 14 & 14 & 12 & 13 & 13 & 13 & 13 & 14 & 0.4 & 0.09 & 0.93 & 0.01 \\
\hline \multicolumn{15}{|l|}{ d 0 to end } \\
\hline Water & 295 & 309 & 320 & 320 & 317 & 294 & 324 & 309 & 323 & 314 & 9.4 & 0.06 & 0.91 & 0.73 \\
\hline Protein & 73 & 76 & 79 & 80 & 79 & 73 & 77 & 77 & 81 & 76 & 2.3 & 0.02 & 0.60 & 0.81 \\
\hline Lipid & 50 & 51 & 51 & 57 & 44 & 47 & 44 & 54 & 53 & 52 & 1.5 & $<0.01$ & 0.69 & $<0.01$ \\
\hline Ash & 11 & 12 & 13 & 13 & 11 & 11 & 12 & 12 & 12 & 13 & 0.4 & 0.01 & 0.80 & 0.09 \\
\hline
\end{tabular}


Table 11

Pairwise comparisons and contrasts of antibiotic supplementation on the chemical body composition of piglets during the carryover period

\begin{tabular}{|c|c|c|c|c|c|c|c|}
\hline \multirow[t]{3}{*}{ Item ${ }^{1}$} & \multicolumn{7}{|c|}{ Pairwise comparisons ${ }^{1}$} \\
\hline & \multicolumn{5}{|c|}{ CP level, \% } & \multicolumn{2}{|c|}{ Contrasts } \\
\hline & 16 & 18 & 20 & 22 & 24 & Linear & Quadratic \\
\hline \multicolumn{8}{|c|}{ Deposition rate, $\mathrm{g} / \mathrm{d}$} \\
\hline \multicolumn{8}{|c|}{ d 14 to end } \\
\hline Water & 0.30 & 0.17 & 0.00 & 0.76 & 0.69 & 0.02 & 0.02 \\
\hline Protein & 0.10 & 0.22 & 0.02 & 0.51 & 0.65 & $<.0 .01$ & $<.0 .01$ \\
\hline Lipid & 0.01 & $<.0 .01$ & 0.01 & 0.07 & 0.01 & 0.02 & 0.02 \\
\hline \multicolumn{8}{|c|}{ d 0 to end } \\
\hline Protein & 0.90 & 0.50 & 0.30 & 0.74 & 0.50 & 0.02 & 0.03 \\
\hline Lipid & 0.31 & $<.0 .01$ & 0.04 & 0.08 & 0.03 & 0.01 & 0.02 \\
\hline Ash & 0.96 & 0.28 & 0.42 & 0.08 & 0.16 & 0.01 & 0.01 \\
\hline
\end{tabular}

\section{Cp Requirements}

According to the model with the minimum AIC, the CP requirement based on ADG was 23.01 and $22.65 \%$, and based on PD was 24.00 and $23.29 \%$ for antibiotic-free diet and antibiotic diet, respectively. The $\mathrm{N}$ intake requirement based on ADG was 16.15 and $16.55 \mathrm{~g} / \mathrm{d}$, and based on PD was 16.27 and $16.36 \mathrm{~g} / \mathrm{d}$ for antibiotic-free diet and antibiotic diet, respectively (Table 12).

Table 12

Requirements of dietary CP level and daily N intake on ADG or PD determined by broken-line and curvilinear-plateau models

\begin{tabular}{|c|c|c|c|c|c|c|c|c|}
\hline \multirow[t]{3}{*}{ Item } & \multicolumn{4}{|c|}{ Antibiotic-free diet } & \multicolumn{4}{|l|}{ Antibiotic diet } \\
\hline & \multicolumn{2}{|c|}{ Broken-line model } & \multicolumn{2}{|c|}{ Curvilinear-plateau model } & \multicolumn{2}{|c|}{ Broken-line model } & \multicolumn{2}{|c|}{ Curvilinear-plateau mode } \\
\hline & Requirement & $\mathrm{AlC}^{1}$ & Requirement & $\mathrm{AlC}^{1}$ & Requirement & $\mathrm{AlC}^{1}$ & Requirement & $\mathrm{AlC}^{1}$ \\
\hline \multicolumn{9}{|c|}{ CP level, \% } \\
\hline$A D G^{2}$ & 23.01 & 25.3 & 42.50 & 26.7 & 20.70 & 16.9 & 22.65 & 10.8 \\
\hline$P D^{3}$ & $24.00^{4}$ & & & & 20.71 & 2.9 & 23.29 & -2.3 \\
\hline \multicolumn{9}{|c|}{ N intake, $g / d$} \\
\hline$A D G^{2}$ & 16.15 & 15.9 & 105.10 & 16.0 & 14.58 & 17.4 & 16.55 & 9.3 \\
\hline $\mathrm{PD}^{3}$ & $16.27^{4}$ & & & & 14.59 & 3.2 & 16.36 & -0.87 \\
\hline \multicolumn{9}{|c|}{${ }^{1} \mathrm{AIC}$, Akaike information criterion } \\
\hline \multicolumn{9}{|c|}{${ }^{2} A D G$, average daily gain } \\
\hline \multicolumn{9}{|c|}{${ }^{3} \mathrm{PD}$, protein deposition } \\
\hline
\end{tabular}

\section{Discussion}

Establishing optimal requirements for $\mathrm{CP}$ is of great importance for maximizing the growth of weaned piglets [15]. However, the current recommendation for dietary CP content in pig was mostly based on the addition of antibiotics in the diet, which may not be able to meet the optimal nutritional demand in the era of banned in-feed antibiotics. Therefore, we investigated the effects of dietary CP level (16, 18, 20, 22, 24\%) and antibiotic supplementations (with or without antibiotics) on growth performance, body composition and tissue deposition. 
We found that the growth performance of piglets during the first two weeks after weaning was significantly improved with increasing dietary CP level in either antibiotic diet or antibiotic-free diet. Moreover, a high-CP diet with or without antibiotics had no effect on post-weaning diarrhea during the treatment period. The results of the present study concerning the growth performance were in consistent with the previous study that dietary $\mathrm{CP}$ level ranging from 17.0 to $23.0 \%$ had a positive effect on newly weaned piglet growth [24]. However, most studies have found that higher protein supply, especially in the absence of in-feed antibiotics, increases the risk of post-weaning diarrhea, which may be due to the increase of microbial fermentation of undigested protein in the gastrointestinal tract that resulted in growth check [25, 26]. Kil and Stein [27] suggested that CP level in piglet diets should be reduced to below $18 \%$ during the immediate post-weaning period when in-feed antibiotic was not used. Our results were also contrary to our previous study [28] conducted in a relatively poor sanitation that an increase in dietary CP level from 17.0 to $23.7 \%$ increased the incidence of post-weaning diarrhea and decreased growth performance of piglets. In that study, the diarrhea incidence of all treatments from $\mathrm{d} 0$ to 14 for the 3-week-weaned pigs ranged from 24.6 to $56.2 \%$, and the ADG ranged from 26 to $107 \mathrm{~g} / \mathrm{d}$, which were far less than the NRC [18] recommendations of $210 \mathrm{~g} / \mathrm{d}$ and $335 \mathrm{~g} / \mathrm{d}$ for piglets from 5 to $7 \mathrm{~kg}$ and from 7 to $11 \mathrm{~kg}$, respectively. The vast difference in the results of the two studies might be explained by the difference in the health status of animals. The enhancement of piglet health and reduction of post-weaning diarrhea in the present study may be due to the piglets being housed in a relatively clean and well-managed farm with a high level of biosecurity. On August 3, 2018, an African swine fever (ASF) occurred in Shenyang, a city in Northeast China [29]. In order to prevent the outbreak of ASF in pigs, the farms in the present study improved biosecurity measures, such as insect and rodent control, thorough cleaning and disinfection of buildings, feed and water hygiene, which improves the health of piglets and reduces the incidences of post-weaning diarrhea. A significant reduction in growth performance was observed in the present study when piglets were fed a low-CP diet. Gloaguen et al. [30] suggested that the supply of $\mathrm{N}$ for the synthesis of dispensable AA may be a limiting factor on pig growth when a sufficient amount of indispensable AA was provided. The dietary protein efficiency of pigs depends on the digestibility of protein and its composition of AA, as well as the content and balance of AA related to animal requirements [31]. Therefore, the response of animals to changes in dietary CP level differ across studies.

In the present study, it was found that antibiotics did not significantly improve piglet growth performance. The results are in contrast to previous research [32-34] where the antibiotic improved piglet performance and reduced diarrhea and mortality. However, our results are consistent with those reported by Holt et al. [35] that antibiotics had little impact on growth performance in clean, isolated facilities with high labor inputs. It is generally accepted that the positive effect of antibiotics is related to its ability to inhibit the growth of certain pathogenic microorganisms [5]. However, the use of antibiotics as growth promoters has been banned in many countries worldwide due to increasing concerns about antibiotic resistance of pathogens, which poses a threat to both human and animal health. Therefore, alternative solutions need to be developed. In fact, efforts in management and nutritional strategies can improve the ability of pigs to prevent the colonization of bacterial pathogens in the gastrointestinal tract and to ameliorate the impact of removing antibiotics from piglet diets $[27,36]$. Pairwise comparisons between antibiotic-free and antibiotic diets at different CP levels except at $18 \% \mathrm{CP}$ revealed no differences in piglet performance, which reflects a high health status and good management of the piglets in the present study. There was no effect of antibiotic supplementation on ADG from d 14 to end of nursery period, which was consistent with the results of Diana et al. [32] and Weber et al. [34] who reported antibiotics had no carryover effects on growth performance. Increasing dietary CP level tended to increase ADG during the carryover period, which was different from those piglets that were severely restricted in nutrient intake during the treatment period and had significant compensatory growth during the re-alimentation period [37,38]. The ability for compensatory gain depends upon the extent, timing and duration of nutrient restriction $[39,40]$.

With the increase of dietary CP level, weights of fasted body, empty body, eviscerated carcass, organs, and individual organ including blood, liver, lungs, kidneys and intestines at $d 14$ were improved. Chen et al. [41] suggested that the increase of organ weight may be the physiological adaptation of pigs to metabolize more protein intake. Although the actual weights of most physical body composition were improved with the increase of CP level, the relative weight was not affected, which was consistent with previous studies [42,43]. Pairwise comparisons of piglets fed diets with or without antibiotics also revealed that antibiotics increased the actual weights of eviscerated carcass and blood on d 14 at $18 \%$ CP, but not relative weight. There was little difference in the proportional weight of physical body composition, which may be due to the fact that it was more closely related to pig genotypes and BW than dietary CP level [44], or that piglets fed treatment diet for a shorter time cannot cause its differences. The early-stage after weaning is recognized as one of the most effective stages for converting nutrients into animal tissues [45]. As expected, the chemical body composition of piglets was heavily influenced by the CP level. Increasing dietary CP level resulted in higher body protein content on d 14 due to linearly improved PD rate during the treatment period, which was consistent with previous studies $[43,46]$. The PD rate is primarily determined by the lean growth potential of pigs [47]. Therefore, low CP supply may severely limit the genetic potential of pigs. Deng et al. [48] reported a reduction in dietary protein content suppressed protein synthesis in liver, pancreas, kidney, and longissimus muscle of piglets, which may be due to the decrease of AA availability and inhibition mTOR signaling. Besides, the synthesis of AA in the body is also influenced by other factors, such as substrate availability, age, physiological and pathological states, gut microbiota, and environmental conditions [49]. Body lipid deposition tended to be decreased, and lipid:protein significantly decreased with the increase of dietary CP level. These findings are in agreement with those reported by Skiba [50] and Morazán et al. [51], who reported pigs fed a low-CP diet with ideal protein profile had fatter carcasses than those fed high-CP diet. A possible reason for these findings is that at low CP level, PD is minimal and a large amount of energy is retained as fat; however, as dietary CP level increases, PD increases and the energy stored as fat decreases [52]. In addition, the observed changes in deposition rates of water and ash caused by CP level reflect the concomitant changes in PD rate, as previous studies have indicated that they are closely related [21,53]. Antibiotics tended to have greater deposition rates of body water and protein coincided with the tendency to have a higher ADG during the treatment period.

Although the physical and chemical body composition slaughtered at a target BW of $25 \mathrm{~kg}$ remained unchanged, deposition rates of body water, protein and lipid during the carryover period as well as deposition rates of protein, lipid and ash during the entire nursery period were improved quadratically as

Page $17 / 21$ 
the CP level increased. The results showed that dietary CP had a significant carryover effect on tissue deposition unlike that on growth performance, indicating that body composition traits were more sensitive to dietary CP level than production traits. No compensatory PD was observed during the carryover period, which consistent with the results of [54, 55]. However, Bikker et al. [56] and Drouillard et al. [57] found nutritional history during the growing period had a compensatory effect on subsequent PD during the finishing period. Martínez-Ramírez et al. [55] suggested that the difference among studies might be attributed to the difference in the upper limit to PD, which is the main factor limiting the extent of compensatory PD. Pairwise comparisons showed that antibiotics increased lipid deposition at high CP levels. In a study of Cho et al. [58], the administration of sub-therapeutic antibiotics increased fat mass in young mice and altered the regulation of hepatic lipids, cholesterol and triglyceride metabolism. Early exposure to antibiotics increases adiposity may be related to the shifts of microbiota to improve short-chain fatty acids production and carbohydrates and energy utilization [59]. However, lower lipid deposition was found for piglets fed diets with antibiotics at low CP levels. This discrepancy between the effect of antibiotics on lipid deposition at low and high CP deserves further exploring.

There are different models to describe the growth response of animals to the nutrient supply, of which the broken-line and curvilinear-plateau models are the most frequently used [60]. Therefore, based on ADG and PD, we used the aforementioned two models to study the CP requirement of piglets.

According to the model with the minimum AIC, the $\mathrm{CP}$ requirement for piglets fed antibiotic-free diet was slightly higher than that of piglets fed antibiotic diet regardless of whether the response criteria were based on ADG (23.01 vs. $22.65 \%)$ or PD (24.00 vs. $23.29 \%)$. It was also found that the CP requirement for maximum PD was higher than for maximum ADG, which was consistent with the results of Batterham et al. (1990) [52]. However, daily $\mathrm{N}$ intake of piglets fed antibiotic-free diet for maximum ADG (16.15 vs. $16.55 \mathrm{~g} / \mathrm{d})$ and PD (16.27 and $16.36 \mathrm{~g} / \mathrm{d})$ were slightly less than those fed antibiotic diet. This indicates that it is important to express the CP requirements of piglets in different ways [61]. In general, however, there was little difference in CP requirements for piglets fed diets with and without antibiotics, which was contrary to our previous hypothesis. These observations confirm once again that antibiotics have little impact on healthy piglets raised under good biosecurity farms. The $\mathrm{CP}$ requirements for piglets fed diets with and without antibiotics were both greater than NRC (2012) with CP level of $22.38 \%$ and N intake of $15.10 \mathrm{~g} / \mathrm{d}$, which was calculated by entering model inputs (average BW during the trial and the NE content in the diets) in NRC (2012) modeling program. The requirements estimated in the present study aligned with the results of Jones et al. [11] (23.80\% CP and $16.79 \mathrm{~g} / \mathrm{d}$ N intake for piglets fed antibiotic-free diet). Both the differences in genetic potential for lean growth rate and differences in health status have a significant impact on the $\mathrm{CP}$ requirements for weaned piglets [62]. It should also be noted that newly weaned pig is in an extremely energy dependent phase of growth and that maintaining proper $\mathrm{N}$ to calorie ratios to maximize their potential for protein deposition is essential [1].

From a practical point of view, reducing the dietary CP level may be extremely important for newly weaned piglets raised in an environment that poses a challenge to pig health. As previously reported, piglets challenged with enterotoxigenic Escherichia coli (ETEC) on the high-CP diet showed a greater decline in performance than those on the low-CP diet [10], and feeding a low-CP diet for 7 or $14 \mathrm{~d}$ after weaning markedly reduced the incidence of postweaning diarrhea after infection with ETEC [26]. However, if piglets are healthy and not suffering from diarrhea, the dietary CP level should be properly increased to enhance the growth potential of the piglets.

\section{Conclusions}

Increasing dietary CP level linearly increased the growth performance and PD of piglets for the first two weeks after weaning, and the carryover effects of treatment diets existed. The CP requirement for piglets with high health status fed antibiotic-free diet was slightly higher than those fed antibiotic diet.

\section{Abbreviations}

AA, amino acids; ADFI, average daily feed intake; ADG, average daily gain; AIC, Akaike information criterion; AOAC, Association of Official Analytical Chemists; ASF, African swine fever; ISG, initial slaughter group; BW, body weight; CP, crude protein; ETEC, enterotoxigenic Escherichia coli; GE, gross energy; $\mathrm{G}$

F, gain:feed; ME, metabolized energy; N, nitrogen; NE, net energy; NRC, National Research Council; PD, protein deposition

\section{Declarations}

\section{Acknowledgements}

Not applicable.

\section{Authors' contributions}

LH, LW and ZYJ conceived and designed research; LH, CZ, XLW, HX, QWW, ZLL, ZKW, YQQ, XFY and KAG participated in acquisition of data, and analysis and/or interpretation of data; LH, LW and ZYJ participated in drafting the manuscript. The authors have read and approved the final manuscript.

\section{Funding}


This research was funded by National Key Research and Development Program of China (2018YFD0501100 and 2016YFD0500501), the Outstanding Talents Training Program of Guangdong Academy of Agricultural Sciences (R2018PY-JC001), the China Agriculture Research System (CARS-35), the Project of Swine Innovation Team in Guangdong Modern Agricultural Research System (2019KJ126).

\section{Availability of data and materials}

The datasets supporting the conclusions of this article are included within the article.

\section{Ethics approval and consent to participate}

The experimental protocols and procedures performed in this study were approved by the Animal Care and Use Committee at Guangdong Academy of Agricultural Sciences.

\section{Consent for publication}

Not applicable.

\section{Competing interests}

The authors declare that they have no competing interests.

\section{Author details}

${ }^{1}$ Institute of Animal Nutrition, Northeast Agricultural University, Harbin 150030, China;

${ }^{2}$ Institute of Animal Science, Guangdong Academy of Agricultural Sciences, State Key Laboratory of Livestock and Poultry Breeding, Ministry of Agriculture Key Laboratory of Animal Nutrition and Feed Science in South China, Guangdong Provincial Key Laboratory of Animal Breeding and Nutrition, Maoming Branch, Guangdong Laboratory for Lingnan Modern Agriculture, Guangzhou 510640, China;

${ }^{3}$ School of Life Science and Engineering, Foshan University, Foshan 528225, China;

${ }^{4}$ College of Animal Science, South China Agricultural University, Guangzhou 510642, China;

\section{References}

1. Pluske JR, Dividich JL, Verstegen MWA. Weaning the pig: Concepts and consequences. Wageningen: Wageningen Acad Publishers; 2003.

2. Kerr BJ, Southern LL, Bidner TD, Friesen KG, Easter RA. Influence of dietary protein level, amino acid supplementation, and dietary energy levels on growing-finishing pig performance and carcass composition. J Anim Sci. 2003;81:3075-87.

3. Parr TM, Kerr BJ, Baker DH. Isoleucine requirement of growing (25 to $45 \mathrm{~kg}$ ) pigs. J Anim Sci. 2003;81:745-52.

4. Warnants N, Van Oeckel MJ, De Paepe M. Study of the optimum ideal protein level for weaned piglets. J Anim Physiol Anim Nutr (Berl). 2001;85:356-68.

5. Cromwell GL. Why and how antibiotics are used in swine production. Anim Biotechnol. 2002;13:7-27.

6. Pi Y, Gao K, Peng Y, Mu CL, Zhu WY. Antibiotic-induced alterations of the gut microbiota and microbial fermentation in protein parallel the changes in host nitrogen metabolism of growing pigs. Animal. 2019;13:262-72.

7. Choi JY, Kim JS, Ingale SL, Kim KH, Shinde PL, Kwon IK, et al. Effect of potential multimicrobe probiotic product processed by high drying temperature and antibiotic on performance of weanling pigs. J Anim Sci. 2011;89:1795-804.

8. Yu M, Zhang C, Yang Y, Mu C, Su Y, Yu K, et al. Long-term effects of early antibiotic intervention on blood parameters, apparent nutrient digestibility, and fecal microbial fermentation profile in pigs with different dietary protein levels. J Anim Sci Biotechnol. 2017;8:60.

9. Wellock IJ, Fortomaris PD, Houdijk JGM, Kyriazakis I. The effect of dietary protein supply on the performance and risk of post-weaning enteric disorders in newly weaned pigs. Anim Sci. 2006;82:327-35.

10. Wellock IJ, Fortomaris PD, Houdijk JGM, Kyriazakis I. Effects of dietary protein supply, weaning age and experimental enterotoxigenic Escherichia coli infection on newly weaned pigs: health. Animal. 2008;2:834-42.

11. Jones CK, Tokach MD, Usry JL, Neill CR, Patience JF. Evaluating lysine requirements of nursery pigs fed low protein diets with different sources of nonessential amino acids. J Anim Sci. 2014;92:3460-70.

12. Nyachoti CM, Omogbenigun FO, Rademacher M, Blank G. Performance responses and indicators of gastrointestinal health in early-weaned pigs fed low-protein amino acid-supplemented diets. J Anim Sci. 2006;84:125-34.

13. Yu D, Zhu W, Hang S. Effects of Long-Term Dietary Protein Restriction on Intestinal Morphology, Digestive Enzymes, Gut Hormones, and Colonic Microbiota in Pigs. Animals. 2019;9:180.

14. Soumeh EA, van Milgen J, Sloth NM, Corrent E, Poulsen HD, Nørgaard JV. Requirement of standardized ileal digestible valine to lysine ratio for 8- to 14-kg pigs. Animal. 2015;9:1312-8. 
15. Zhou H, Chen DW, Mao X, He J, Yu J, Zheng P, et al. Evaluation of standardized ileal digestible lysine requirement for 8-20 kg pigs fed low crude protein diets. Anim Sci J. 2018;90:1-10.

16. Morise A, Seve B, Mace K, Magliola C, Le Huerou-Luron I, Louveau I. Growth, body composition and hormonal status of growing pigs exhibiting a normal or small weight at birth and exposed to a neonatal diet enriched in proteins. Br J Nutr. 2011;105:1471-9.

17. Morise A, Sève B, Macé K, Magliola C, Le Huërou-Luron I, Louveau I. Impact of Intrauterine Growth Retardation and Early Protein Intake on Growth, Adipose Tissue, and the Insulin-Like Growth Factor System in Piglets. Pediatr Res. 2008;65:45-50.

18. NRC. Nutrient Requirements of Swine. 11th ed. Washington: Natl Acad Press; 2012.

19. Jones CK, Gabler NK, Main RG, Patience JF. Characterizing growth and carcass composition differences in pigs with varying weaning weights and postweaning performance. J Anim Sci. 2012;90:4072-80.

20. AOAC. Official methods of analysis. 17th ed. Arlington: Association of Official Analytical Chemists; 2000.

21. Colina JJ, Miller PS, Lewis AJ, Fischer RL, Diedrichsen RM. Body composition, tissue deposition, and lysine utilization for protein deposition of barrows and gilts fed crystalline or protein-bound lysine. J Anim Sci. 2016;94:1972-81.

22. Robbins KR, Saxton AM, Southern LL. Estimation of nutrient requirements using broken-line regression analysis. J Anim Sci. 2006;84:E155-65.

23. Burnham KP, Anderson DR. Model Selection and Multimodel Inference: a practical information-theoretic approach. 2nd ed. New York: SpringerVerlag; 2002.

24. Opapeju FO, Rademacher M, Blank G, Nyachoti CM. Effect of low-protein amino acid-supplemented diets on the growth performance, gut morphology, organ weights and digesta characteristics of weaned pigs. Animal. 2008;2:1457-64.

25. Heo JM, Kim JC, Hansen CF, Mullan BP, Hampson DJ, Pluske JR. Effects of feeding low protein diets to piglets on plasma urea nitrogen, faecal ammonia nitrogen, the incidence of diarrhoea and performance after weaning. Arch Anim Nutr. 2008;62:343-58.

26. Heo JM, Kim JC, Hansen CF, Mullan BP, Hampson DJ, Pluske JR. Feeding a diet with decreased protein content reduces indices of protein fermentation and the incidence of postweaning diarrhea in weaned pigs challenged with an enterotoxigenic strain of Escherichia coli. $J$ Anim Sci. 2009;87:2833-43.

27. Kil DY, Stein HH. Invited Review: Management and feeding strategies to ameliorate the impact of removing antibiotic growth promoters from diets fed to weanling pigs. Can J Anim Sci. 2010;90:447-60.

28. Wu YP, Jiang ZY, Zheng CT, Wang L, Zhu C, Yang XF, et al. Effects of protein sources and levels in antibiotic-free diets on diarrhea, intestinal morphology, and expression of tight junctions in weaned piglets. Anim Nutr. 2015;1:170-6.

29. Zhou X, Li N, Luo Y, Liu Y, Miao F, Chen T, et al. Emergence of African Swine Fever in China, 2018. Transbound Emerg Dis. 2018;65:1482-4.

30. Gloaguen M, Le Floc'h N, Corrent E, Primot Y, van Milgen J. The use of free amino acids allows formulating very low crude protein diets for piglets. J Anim Sci. 2014;92:637-44.

31. van Milgen J, Dourmad J-Y. Concept and application of ideal protein for pigs. J Anim Sci Biotechnol. 2015;6:15.

32. Diana A, Boyle LA, Leonard FC, Carroll C, Sheehan E, Murphy D, et al. Removing prophylactic antibiotics from pig feed: how does it affect their performance and health? BMC Vet Res. 2019;15:67.

33. Kyriakis SC, Bourtzi-Hatzopoulou E, Alexopoulos C, Kritas SK, Polyzopoulou Z, Lekkas S, et al. Field evaluation of the effect of in-feed doxycycline for the control of ileitis in weaned piglets. J Vet Med B. 2002;49:317-21.

34. Weber TE, Schinckel AP, Houseknecht KL, Richert BT. Evaluation of conjugated linoleic acid and dietary antibiotics as growth promotants in weanling pigs. J Anim Sci. 2001;79:2542-9.

35. Holt JP, van Heugten E, Graves AK, See MT, Morrow WEM. Growth performance and antibiotic tolerance patterns of nursery and finishing pigs fed growth-promoting levels of antibiotics. Livest Sci. 2011;136:184-91.

36. Li J. Current status and prospects for in-feed antibiotics in the different stages of pork production - A review. Asian-Australas J Anim Sci. 2017;30:1667-73.

37. Shi Q, Zhu Y, Wang J, Yang H, Wang J, Zhu W. Protein restriction and succedent realimentation affecting ileal morphology, ileal microbial composition and metabolites in weaned piglets. Animal. 2019;13:2463-72.

38. Zhao X, Fu HY, Qiu SN, Teng T, Bai GD, Ju DX, et al. Effects of early protein restriction on the growth performance and gut development of pigs fed diets with or without antibiotic. Animal. 2019;24:1-10.

39. Chaosap C, Parr T, Wiseman J. Effect of compensatory growth on performance, carcass composition and plasma IGF-1 in grower finisher pigs. Animal. 2011;5:749-56.

40. Lebret B, Heyer A, Gondret F, Louveau I. The response of various muscle types to a restriction -re-alimentation feeding strategy in growing pigs. Animal. 2007;1:849-57.

41. Chen HY, Miller P, Lewis AJ, Wolverton C, Stroup W. Changes in plasma urea concentration can be used to determine protein requirements of two populations of pigs with different protein accretion rate. J Anim Sci. 1995;73:2631-9.

42. Bellego L, Noblet J. Performance and utilization of dietary energy and amino acids in piglets fed low protein diets. Livest Prod Sci. 2002;76:45-58.

43. Conde-Aguilera J, Aguinaga MA, Lara L, Aguilera J, Nieto R. Carcass traits and organ weights of 10-25-kg body weight Iberian pigs fed diets with different protein-to-energy ratio. Anim Feed Sci Technol. 2011;164:116-24.

Page 20/21 
44. de Lange CFM, Morel PCH, Birkett SH. Modeling chemical and physical body composition of the growing pig. J Anim Sci. 2003;81:E159-65.

45. Kendall DC, Gaines AM, Allee GL, Usry JL. Commercial validation of the true ileal digestible lysine requirement for eleven- to twenty-seven-kilogram pigs1. J Anim Sci. 2008;86:324-32.

46. Ruiz-Ascacibar I, Stoll P, Kreuzer M, Boillat V, Spring P, Bee G. Impact of amino acid and CP restriction from 20 to $140 \mathrm{~kg}$ BW on performance and dynamics in empty body protein and lipid deposition of entire male, castrated and female pigs. Animal. 2016;11:394-404.

47. Ruusunen M, Partanen K, Pösö R, Puolanne E. The effect of dietary protein supply on carcass composition, size of organs, muscle properties and meat quality of pigs. Livest Sci. 2007;107:170-81.

48. Deng D, Yao K, Chu W, Li T, Huang R, Yin Y, et al. Impaired translation initiation activation and reduced protein synthesis in weaned piglets fed a lowprotein diet. J Nutr Biochem. 2009;20:544-52.

49. Wu G, Wu Z, Dai Z, Yang Y, Wang W, Liu C, et al. Dietary requirements of "nutritionally non-essential amino acids" by animals and humans. Amino Acids. 2013;44:1107-13.

50. Skiba G. Physiological aspects of compensatory growth in pigs. J Anim Feed Sci. 2005;14:191-203.

51. Morazán H, Álvarez-Rodríguez J, Seradj A, Balcells J, Babot D. Trade-offs among growth performance, nutrient digestion and carcass traits when feeding low protein and/or high neutral-detergent fiber diets to growing-finishing pigs. Anim Feed Sci Technol. 2015;207:168-80.

52. Batterham E, Andersen L, Baigent D, White E. Utilization of ileal digestible amino acids by growing pigs: Effect of dietary lysine concentration on efficiency of lysine retention. Br J Nutr. 1990;64:81-94.

53. Gómez R, Lewis AJ, Miller P, Chen HY, Diedrichsen R. Body composition and tissue accretion rates of barrows fed corn-soybean meal diets or lowprotein, amino acid-supplemented diets at different feeding levels. J Anim Sci. 2002;80:654-62.

54. Ferguson N, Theeruth B. Protein and lipid deposition rates in growing pigs following a period of excess fattening. S Afr J Anim Sci. 2002;32:97105.

55. Martínez-Ramírez H, Jeaurond E, de Lange C. Dynamics of body protein deposition and changes in body composition after sudden changes in amino acid intake: I. Barrows. J Anim Sci. 2008;86:2156-67.

56. Bikker P, Verstegen M, Kemp B, Bosch M. Performance and Body Composition of Finishing Gilts (45 to 85 Kilograms) as Affected by Energy Intake and Nutrition in Earlier Life: I. Growth of the Body and Body Components. J Anim Sci. 1996;74:806-16.

57. Drouillard J, Britton R, Bauer M, Gramlich S, Wester T, Ferrell C. Growth, body composition, and visceral organ mass and metabolism in lambs during and after metabolizable protein or net energy restrictions. J Anim Sci. 1991;69:3357-75.

58. Cho I, Yamanishi S, Cox L, Methe BA, Zavadil J, Li K, et al. Antibiotics in early life alter the murine colonic microbiome and adiposity. Nature. 2012;488:621-6.

59. Che L, Hu Q, Wang R, Zhang D, Liu C, Zhang Y, et al. Inter-correlated gut microbiota and SCFAs changes upon antibiotics exposure links with rapid body-mass gain in weaned piglet model. J Nutr Biochem. 2019;74:108246.

60. Pomar C, Kyriazakis I, Emmans G, Knap P. Modeling stochasticity: Dealing with populations rather than individual pigs. J Anim Sci. 2003;81:E17886.

61. Elsbernd AJ, de Lange CFM, Stalder KJ, Karriker LA, Patience JF. SID lysine requirement of immunologically and physically castrated male pigs during the grower, early and late finisher periods. J Anim Sci. 2017;95:1253-63.

62. NRC. Nutrient Requirements of Swine. 10th ed. Washington: Natl Acad Press; 1998. 